\title{
Effect of Section and Compression of the Optic Nerve on the ERG in the Rabbit
}

\author{
By \\ Nobuhiro Abe \\ Department of Ophthalmology, School of Medicine, Tohoku University \\ Sendai; Director: Prof. N. Kirisawa
}

(Received for publication, September 20, 1962)

\section{INTRODUCTION}

Jacobson and Gestring ${ }^{1)}$ observed that the amplitude of the ERG increased following optic nerve section in cat and monkey and that section of the optic nerve eliminated the effects of drugs on the ERG; they thus concluded that the optic nerve contains some centrifugal fibers. Granit ${ }^{2)}$, Dodt ${ }^{3)}$, Wolter $^{4}$, Polyak $^{5}$, Motokawa \& Ebe $^{67}$, Mita ${ }^{7}$ and Ogawa ${ }^{8}$ have also reported the possibility of central control of retinal function by centrifugal fibers, but Arden ${ }^{9)}$ had argued against the existence of such centrifugal fibers. The present paper reports on changes in the ERG of rabbits in the first 12 hours following section or compression of the optic nerve, using the amplitude of the b-wave as an index.

\section{METHOD}

The optic nerve of rabbits was exposed and the animals were then allowed at least 2 hours for recovery from anesthesia and surgical trauma. Then one of the following operations was performed:

1) Intracranial optic nerve section

2) Intraorbital optic nerve section

3) Intracranial optic nerve compression

4) Intraorbital optic nerve compression

In 1) and 3) the dura mater was gently detached from the orbital wall, taking can to avoid damage to the frontal lobe, and the optic nerve and the $A$. ophthalmica interna which run side by side were separated just before the entrance of the former into the optic canal. In 2) and 4), special care was taken to avoid injuring the A. ciliaris nasalis, in exposing the optic nerve. but the A. centralis retinae was not separated, for the presence of this artery had little, if any, influences on the results. The section of the optic nerve was effected by a sharp stroke with a Graefe's knife, and compression was achieved by powerful pressure upon an

阿部 语轰 
open silver tube placed over the nerve. Light stimuli were provided from a projection bulb with a tungusten filament and flash duration was controlled by a mechanical shutter. After dark adaptation of 1 hour, three flashes of white light (1/2-1/5 sec. duration) were given at $20 \mathrm{sec}$. intervals to obtain the mean amplitude, and the light intensity was adjusted to about 50 lux for the eye. The recording electrode was a contact lens electrode with an $\mathrm{Ag}-\mathrm{AgCl}$ plate and the indifferent electrode was a needle inserted beneath the skin over the cranium. Recordings were made with a CR coupled amplifier (time constant $0.5 \mathrm{sec}$.) and an ink writing oscillograph.

\section{RESULTS}

1) Following intracranial optic nerve section, the amplitude of the b-wave immediately began to increase, in one case, it rose to $200 \%$ in 1.5 hours and to a maximum of $330 \%$ of original value after 6 hours (see Fig. 1). In another case, it reached $200 \%$ of the control value after one half hour and attained a maximum of $330 \%$ within 3 hours. In both cases, the amplitude slowly decreased after the maximum and reached approximately $200 \% 12$ hours after sectioning. The basis for the different time courses in both cases was not investigated.
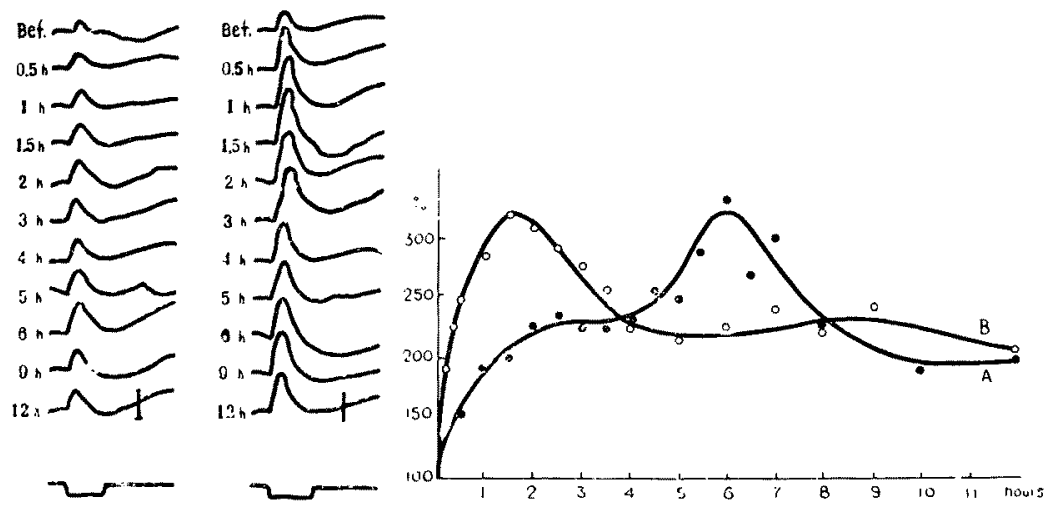

Fig. 1. Effect of intracranial optic nerve section upon rabbit ERG

Left: Two sets of records were obtained from two different animals.

Top records are control. Time after sectioning is given on left of each record.

Downward deflections of bottom traces indicate stimulus (duration $0.2 \mathrm{sec}$.)

Calibration: $0.5 \mathrm{mV}$

Right: A and B refer to left and right series of records respectively.

Abscissa: time after optic nerve section

Ordinate: relative amplitude in \% (control, 100\%)

2) Intraorbital optic nerve section caused a temporary decrease of the amplitude of the b-wave immediately after sectioning, but recovery to the original amplitude occurred in $45 \mathrm{~min}$, thereafter the amplitude showed a gradual 

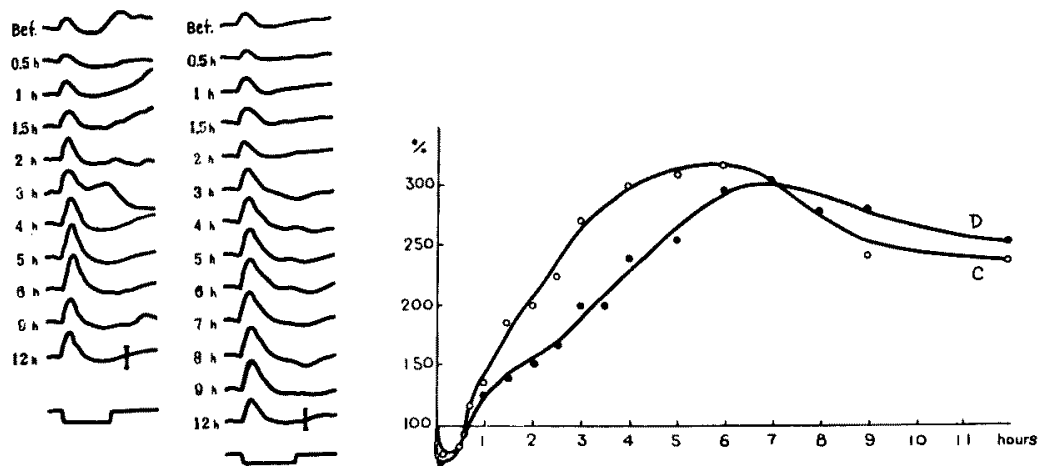

Fig. 2. Effect of intraorbital optic nerve section upon rabbit ERG

$\mathrm{C}$ and $\mathrm{D}$ refer to left and right series of records respectively.

Flash duration: 0.3 sec.

Otherwise as in Fig. 1.

increase, reaching $200 \%$ of the control value in $2-3$ hours and maximum of $300 \%$ in 6-7 hours (see Fig. 2). The subsequent decrease in amplitude was rather slow, a level of $250 \%$ still being maintained 12 hours after sectioning. The initial temporary drop of the $b$-wave amplitude may be attributed to transient circulatory disturbance in the retina due to the inevitable injury of blood vessels.

3) Following intracranial optic nerve compression, the change in the amplitude of the $b$-wave was slow, rising to $200 \%$ in 4 hours and reaching a maximum of $250 \%$ in 6-7 hours; it showed little decrease until 12 hours after compression (see Fig. 3).

4) After intraorbital optic nerve compression, the increase of amplitude of the b-wave was again slow; the maximum amplitude of about $180 \%$ occurred
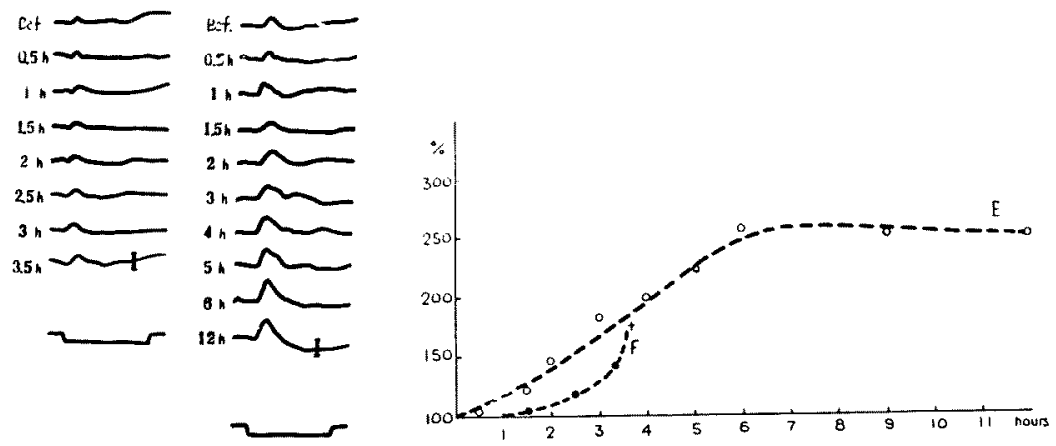

Fig. 3. Effect of intracranial optic nerve compression upon rabbit ERG

$\mathrm{F}$ and $\mathbf{E}$ refer to left and right series of records respectively.

Flash duration: 0.5 sec.

Otherwise as in Fig. 1. 

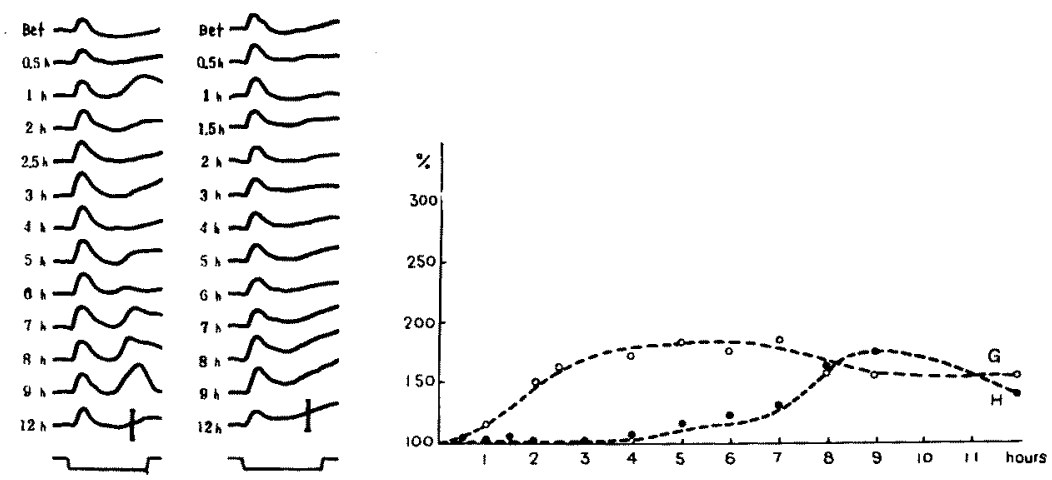

Fig. 4. Effect of intraorbital optic nerve compression upon rabbit ERG $G$ and $H$ refer to left and right series of records respectively. Flash duration: 0.5 sec. Otherwise as in Fig. 1.

after 6-9 hours (see Fig. 4).

\section{COMMENT}

Even when blood vessel damage was minimized, an increase in the amplitude of the b-wave of the ERG occurred immediately following section or compression of the optic nerve. In this series of experiments, sufficient care was taken to eliminate the adverse effects of surgical trauma and anesthesia by allowing at least 2 hours for recovery after exposure of the optic nerve, and then nerve section or compression was made. Therefore, it may be concluded that the changes of the amplitude of the b-wave were a direct result of the lesion in the optic nerve. There are, however, many points of question on the mechanism of such changes. It may be possible that the changes in ERG are due to the interruption of centrifugal control caused by the effect of surgical operation on the optic nerve, as Jacobson and Gestring claim. But it seems also possible that the changes are the result of a secondary metabolic anomaly in the retina caused by the surgical operation on the optic nerve. The results of the present experiments are in line with those by Jacobson et al., but not enough to justify their hypothesis.

\section{SUMMARY}

1) The amplitude of the b-wave of the ERG in rabbits was found to increase following optic nerve section or compression.

2) After section of the optic nerve, either intracranial or intraorbital, the b-wave amplitude rose rapidly, up to the peak of $300-330 \%$ of the original amplitude, but hence decreased to $200-250 \%$ in 12 hours after the lesion of the optic nerve. 
3) After compression of the optic nerve, the rise of the b-wave amplitude was slower. The peak value did not exceed the range of $180-250 \%$, the subsequent drop being also less marked.

4) The results showed no obvious difference whether the site of lesion on the optic nerve was intracranial or intraorbital.

5) The results of the present experiments are in line with those by Jacobson et al., but not enough to justify their hypothesis.

The author indebted to Prof. N. Kirisawa for his continued direction, and to Prof. K. Motokawa and Assist. Prof. K. Tasaki for their invalnable discussion and suggestion throughout the course of the experiment and the preparation of the manuscript.

\section{References}

1) Jacobson, J.H. \& Gestring, G.F., A.M.A. Arch. Ophthal., 1958, 60, 295.

2) Granit, R., J. Neurophysiol., 1955, 18, 387.

3) Dodt, E., J. Neurophysiol., 1956, 19, 301.

4) Wolter, J.R., Graefes Arch. Ophthal., 1957, 158, 524.

5) Polyak, S.L., The University of Chicago Press. 1957, 246.

6) Motokawa, K. \& Ebe, M., J. Neurophysiol., 1954, 17, 364.

7) Mita, T., J. Iwate med. Ass. (Jap.), 1961, 13, 1313.

8) Ogawa, T., J. Iwate med. Ass. (Jap.), 1961, 13, 922.

9) Arden, G., Granit, R. \& Ponte, F., J. Neurophysiol., 1960, 23, 305. 